\title{
Remaining Useful Life Estimation Based on Asynchronous Multisource Monitoring Information Fusion
}

\author{
Yanyan Hu, Shuai Qi, Xiaoling Xue, and Kaixiang Peng \\ Beijing Engineering Research Center of Industrial Spectrum Imaging, School of Automation and Electrical Engineering, \\ University of Science and Technology Beijing, Beijing 100083, China
}

Correspondence should be addressed to Kaixiang Peng; kaixiang@ustb.edu.cn

Received 6 May 2017; Accepted 10 July 2017; Published 13 August 2017

Academic Editor: Adel Haghani

Copyright (C) 2017 Yanyan Hu et al. This is an open access article distributed under the Creative Commons Attribution License, which permits unrestricted use, distribution, and reproduction in any medium, provided the original work is properly cited.

\begin{abstract}
An asynchronous RUL fusion estimation algorithm is presented for the hidden degradation process with multiple asynchronous monitoring sensors based on multisource information fusion. Firstly, a state-space type model is established by modeling the stochastic degradation as a Wiener process and transforming asynchronous indirectly observations in the fusion period to the fusion time. The statistical characteristics of involved noises and their correlations are analyzed. Secondly, the estimate of the hidden degradation state is obtained by applying Kalman filtering with correlated noises to the established state-space model, where the synchronized observations are fused. Also, the unknown model parameters are recursively identified based on the Expectation-Maximization (EM) algorithm with the Generic Algorithm (GA) adopted to solve the maximization problem. Finally, the probability distribution of RUL is obtained using the fused degradation state estimation and the updated identification result of the model parameters. Simulation results show that the proposed fusion method has better performance than the RUL estimation with single sensor.
\end{abstract}

\section{Introduction}

Modern engineering systems are becoming large in scale, huge in investment, and more and more sophisticated in structure with the development of science and technology. As a result, once an accident occurs in these systems, it would cause tremendous damage and enormous loss of property [1]. As time goes on, degradation of equipment in systems will inevitably occur due to complex operating environment and many other reasons, which leads to the reduction of system reliability and safety $[2,3]$. Consequently, it is of great importance to predict the RUL of the system components in order to correctly evaluate the health state of the system and make appropriate maintenance plans to ensure the safe operation of the system and increase economic returns.

The complexity of the system itself and the running environment makes it hard to establish a mechanism model for RUL estimation. In recent years, the RUL estimation based on monitoring data attracts a lot of research attentions due to its wide application range and the ability to quantify the uncertainties of the estimation results $[4,5]$. Si et al. in [6] reviewed the statistical data driven approaches for RUL estimation. The existing methods were classified into two categories: direct condition monitoring data based approaches and indirect condition monitoring data based approaches, which can be further divided into stochastic filtering based methods [7-10], covariance based hazard model methods [11, 12], Wiener-process-based methods [13, 14], Gamma process based methods [15, 16], and Markovian-based methods [17] and others.

Although there are fruitful researches on RUL estimation based on stochastic modeling with monitoring data, most of works are specific to single sensor. However, the complexity of the system itself and the running environment makes the RUL prediction have large uncertainties. At the same time, the information from single source tends to be quite limited. In response to the above issues, multiple sensors are usually utilized to monitor the condition of the system in order to reduce the uncertainties of the system and improve the accuracy of the RUL estimation [3]. Wei et al. in [18] proposed a multisensor information based Remaining Useful Life estimation method with anticipated performance for a 


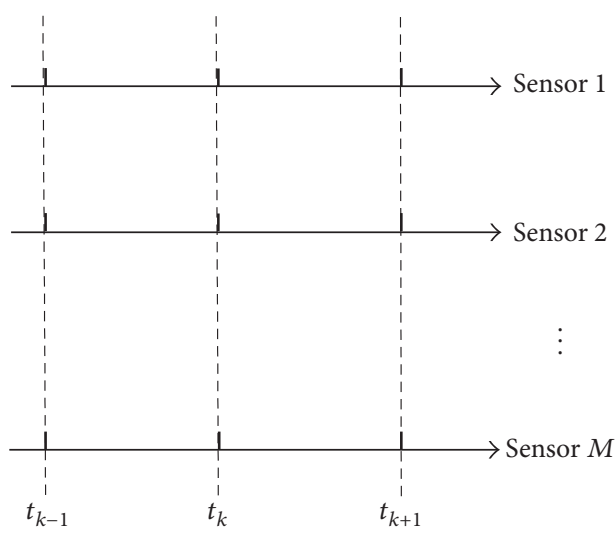

(a) Synchronous

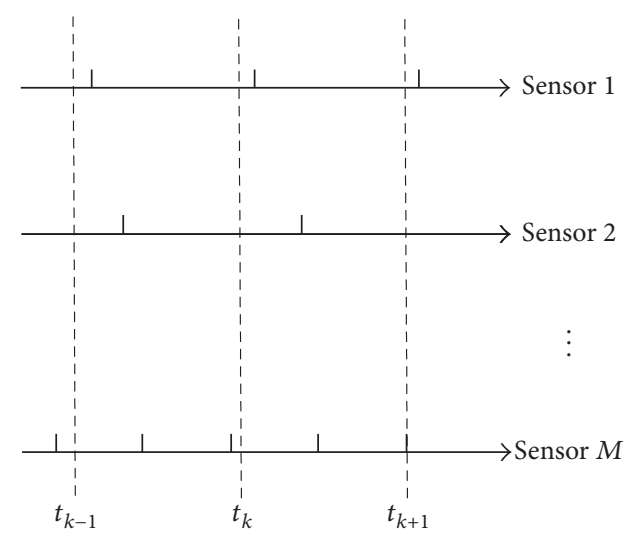

(b) Asynchronous

FIgURE 1: Observations from multiple sensors.

kind of stochastic linear degradation with Wiener properties. The degradation process as well as the RUL distribution with a random threshold was identified recursively by adopting the distributed fusion filtering and a two-stage parameter estimation approach. Meanwhile, the sensor selection problem was also discussed by quantitatively analyzing the benefits of the fusion manipulation. Dong and He in [19] presented a hidden semi-Markov-model based methodology for multisensor equipment health diagnosis and prognosis. Sensor fusion was implemented by adjusting the weight assigned to each sensor based on the discriminant function analysis. A new health indicator was constructed in [20] by fusing the mutual information of multiple features extracted from the vibration signal to predict the RUL of Machinery. Li and He in [21] proposed a methodology to predict the RUL of both wheels and trucks by fusing data from three types of detectors, including wheel impact load detector, machine vision systems, and optical geometry detectors. Although multisource information is used in the above works to improve the performance of RUL estimation, observations from multiple sensors were assumed to be collected at the same time, that is, time consistent as shown in Figure 1(a). However, in practical engineering, observations from multiple sensors are usually not aligned in time due to different sampling periods, distinct initial sampling time, and many other reasons $[22,23]$. This results in that multisensor observations are not synchronous but asynchronous as shown in Figure 1(b) and poses new challenges to the RUL estimation.

Consequently, motivated by the above discussions, the asynchronous RUL fusion estimation problem is studied in this paper. We assume that the hidden degradation which is modeled as Wiener process with unknown model parameters is observed by an arbitrary number of asynchronous sensors, whose sampling frequencies and initial sampling times are all arbitrary. The asynchronous sensor observations are firstly synchronized to the fusion time and then fused using the Kalman filtering technology to get the fused estimate of the latent degradation state with the correlations between various noises introduced by the synchronization process analyzed. The unknown model parameters are also recursively identified using the synchronized observations based on the $E M$ algorithm with the maximization problem solved by GA. Finally, the RUL is derived based on the fused estimates of the latent degradation state and unknown model parameters and the simulation example is provided to demonstrate the feasibility and effectiveness of the proposed algorithm.

The organization of this paper is as follows. The fusion estimation problem of RUL for stochastic degradation process with multiple asynchronous sensors is formulated in Section 2. The proposed asynchronous RUL fusion estimation algorithm is derived in Section 3. Section 4 gives simulation results and conclusions are drawn in Section 5.

\section{Problem Formulation}

Wiener process is widely used to model the stochastic degradation of a system attributed to its good mathematical characteristics such as the infinite separability. In general, a linear Wiener-process-based degradation model can be represented as

$$
X(t)=\phi+\eta t+\sigma B(t),
$$

where $X(t)$ is the random variable representing the degradation at time $t, \phi$ is the initial degradation state, $\eta$ and $\sigma$ are, respectively, the drift and diffusion coefficient, and $B(t)$ is the standard Brownian motion reflecting the stochastic dynamics of the degradation process.

We assume the degradation process (1) is observed by a number of $M$ asynchronous sensors. Denote $t_{k-1}$ and $t_{k}$, respectively, as the previous and oncoming fusion time instants. Since these asynchronous sensors may have different sampling periods and the sampling intervals may even be nonuniform, more than one measurement could probably be obtained by a given sensor during the fusion interval $\left(t_{k-1}, t_{k}\right]$. Denote $t_{k}^{m}$ as the time when the latest measurement $y_{k}^{m}$, which is the nearest to $t_{k}$, is observed by sensor $m$, where $y_{k}^{m}$ is the indirect observation of the degradation process described by

$$
y_{k}^{m}=h^{m} x\left(t_{k}^{m}\right)+v\left(t_{k}^{m}\right)
$$

where $x\left(t_{k}^{m}\right)$ is the realization of $X(t)$ at time $t_{k}^{m}$ and $v\left(t_{k}^{m}\right) \sim$ $N\left(0, r^{m}\right)$ is the observation noise. 
Consequently, the objective of this paper is to predict the distribution of RUL of degradation process (1) based on the cumulative measurements $Y^{k}=\left\{y_{k^{\prime}}^{i}, k^{\prime}=1, \ldots, k, i=\right.$ $1, \ldots, M\}$, where the RUL of a system at time $t_{k}$ is defined as

$$
\ell_{k}=\inf \left\{\ell_{k}: X\left(t_{k}+\ell_{k}\right) \geq \omega \mid X(0)<\omega\right\},
$$

where $\omega$ is the predefined threshold level.

\section{The Proposed RUL Fusion Estimation Algorithm}

3.1. Fusion Estimation of the Degradation State. From the degradation process (1), we know that the transition of the degradation state satisfies

$$
x\left(t_{j}\right)=x\left(t_{i}\right)+\eta\left(t_{j}-t_{i}\right)+w\left(t_{j}, t_{i}\right),
$$

where $w\left(t_{j}, t_{i}\right)=\sigma\left(B\left(t_{j}\right)-B\left(t_{i}\right)\right) \sim N\left(0, \sigma^{2}\left(t_{j}-t_{i}\right)\right)$. By substituting (4) into (2) with $t_{j}$ and $t_{i}$ in (4) replaced with $t_{k}$ and $t_{k}^{m}$, respectively, it follows that

$$
\begin{aligned}
y_{k}^{m} & =h^{m} x\left(t_{k}\right)-h^{m} \eta\left(t_{k}-t_{k}^{m}\right)-h^{m} w\left(t_{k}, t_{k}^{m}\right)+v\left(t_{k}^{m}\right) \\
& =h^{m} x\left(t_{k}\right)-h^{m} \eta\left(t_{k}-t_{k}^{m}\right)+\xi_{k}^{m},
\end{aligned}
$$

$$
\begin{aligned}
\operatorname{cov}\left\{v_{k}\right\} & =\left[\begin{array}{llll}
r_{j}{ }^{1} & & \\
& \ddots & \\
& r_{j}{ }^{M}
\end{array}\right], \\
\operatorname{cov}\left(w_{k}\right) & =\left[\begin{array}{cccc}
h^{1} h^{1} \sigma^{2}\left(t_{k}-t_{k}{ }^{1}\right) & h^{1} h^{2} \sigma^{2}\left(t_{k}-t_{k}{ }^{2}\right) & \cdots & h^{1} h^{M} \sigma^{2}\left(t_{k}-t_{k}{ }^{M}\right) \\
h^{2} h^{1} \sigma^{2}\left(t_{k}-t_{k}{ }^{2}\right) & h^{2} h^{2} \sigma^{2}\left(t_{k}-t_{k}{ }^{2}\right) & \cdots & h^{2} h^{M} \sigma^{2}\left(t_{k}-t_{k}{ }^{M}\right) \\
\vdots & \vdots & \vdots & \vdots \\
h^{M} h^{1} \sigma^{2}\left(t_{k}-t_{k}{ }^{M}\right) & h^{M} h^{2} \sigma^{2}\left(t_{k}-t_{k}{ }^{M}\right) & \cdots & h^{M} h^{M} \sigma^{2}\left(t_{k}-t_{k}{ }^{M}\right)
\end{array}\right] .
\end{aligned}
$$

where $\xi_{k}^{m}=v\left(t_{k}^{m}\right)-h^{m} w\left(t_{k}, t_{k}^{m}\right)$. Then, by defining $y_{k}=$ $\left[y_{k}^{1}, \ldots, y_{k}^{M}\right]^{T}, \xi_{k}=\left[\xi_{k}^{1}, \ldots, \xi_{k}^{M}\right]^{T}, h=\left[h^{1}, \ldots, h^{M}\right]^{T}$, and $v_{k}=\left[v\left(t_{k}^{1}\right), \ldots, v\left(t_{k}^{M}\right)\right]^{T}$, the asynchronous indirect observations $\left\{y_{k}^{1}, y_{k}^{2}, \ldots, y_{k}^{M}\right\}$ collected from $M$ asynchronous sensors in $\left(t_{k-1}, t_{k}\right]$ can be regarded as an augmented measurement at $t_{k}$ with

$$
y_{k}=h x\left(t_{k}\right)-\eta T_{k} h+\xi_{k}
$$

where

$$
\begin{gathered}
T_{k}=\left[\begin{array}{ccc}
t_{k}-t_{k}^{1} & & \\
& \ddots & \\
& & t_{k}-t_{k}^{M}
\end{array}\right], \\
\xi_{k}=v_{k}+\left[\begin{array}{c}
h^{1} w\left(t_{k}, t_{k}^{1}\right) \\
\vdots \\
h^{M} w\left(t_{k}, t_{k}^{M}\right)
\end{array}\right]
\end{gathered}
$$

and correspondingly

$$
\operatorname{cov}\left\{\xi_{k}\right\}=\operatorname{cov}\left\{v_{k}\right\}+\operatorname{cov}\left\{w_{k}\right\}
$$

with
Obviously, we see that $\operatorname{cov}\left\{\xi_{k}\right\}$ is a function of parameters $\left\{\sigma^{2}, r^{m}, h^{m}\right\}$, and we denote it as $\operatorname{cov}\left\{\xi_{k}\right\}=\Sigma_{k}\left(\sigma^{2}, r^{m}, h^{m}\right)$ for simplicity.

From (4), we can directly have

$$
x\left(t_{k}\right)=x\left(t_{k-1}\right)+\eta\left(t_{k}-t_{k-1}\right)+w\left(t_{k}, t_{k-1}\right) .
$$

It follows that

$$
\begin{aligned}
\operatorname{cov} & \left\{w\left(t_{k}-t_{k-1}\right), \xi_{k}^{m}\right\} \\
& =\operatorname{cov}\left\{w\left(t_{k}-t_{k-1}\right),-h^{m} w\left(t_{k}-t_{k}^{m}\right)\right\} \\
& =-h^{m} \sigma^{2}\left(t_{k}-t_{k}^{m}\right),
\end{aligned}
$$


Firstly, the predicted estimate and its error covariance are given by

$$
\begin{aligned}
& \hat{x}_{k \mid k-1}=E\left\{x\left(t_{k}\right) \mid Y^{k-1}\right\}=\widehat{x}_{k-1 \mid k-1}+\eta\left(t_{k}-t_{k-1}\right), \\
& P_{k \mid k-1}=\operatorname{var}\left\{x\left(t_{k}\right) \mid Y^{k-1}\right\}=P_{k-1}+\sigma^{2}\left(t_{k}-t_{k-1}\right) .
\end{aligned}
$$

Secondly, the updated estimate and its error covariance are got by fusing the asynchronous indirect observations $y_{k}=$ $\left[y_{k}^{1}, \ldots, y_{k}^{M}\right]^{T}$ in $\left(t_{k-1}, t_{k}\right]$ as

$$
\begin{aligned}
& \widehat{x}_{k}=\widehat{x}_{k \mid k-1}+P_{k}\left[h-\frac{\sigma^{2} T_{k} h}{P_{k \mid k-1}}\right]^{T}\left(M_{k}\left(\sigma^{2}, h^{m}, r^{m}\right)\right)^{-1}\left(y_{k}-h \widehat{x}_{k \mid k-1}+\eta T_{k} h\right), \\
& P_{k}=\frac{1}{1 / P_{k \mid k-1}+\left[h-\sigma^{2} T_{k} h / P_{k \mid k-1}\right]^{T}\left(M_{k}\left(\sigma^{2}, h^{m}, r^{m}\right)\right)^{-1}\left[h-\sigma^{2} T_{k} h / P_{k \mid k-1}\right]^{T}},
\end{aligned}
$$

where $\widehat{x}_{k}=E\left\{x\left(t_{k}\right) \mid Y^{k}\right\}, P_{k}=\operatorname{var}\left\{x\left(t_{k}\right) \mid Y^{k}\right\}$, and

$$
\begin{aligned}
& M_{k}\left(\sigma^{2}, h^{m}, r^{m}\right)=\operatorname{cov}\left\{\xi_{k}\right\} \\
& -\frac{\left(\operatorname{cov}\left\{w\left(t_{k}-t_{k-1}\right), \xi_{k}\right\}\right)^{T}\left(\operatorname{cov}\left\{w\left(t_{k}-t_{k-1}\right), \xi_{k}\right\}\right)}{P_{k \mid k-1}} .
\end{aligned}
$$

Remark 1. Note from (8), (9), and (12) that we know that $M$ is also a function of unknown parameters $\left\{\sigma^{2}, h^{m}, r^{m}\right\}$.

3.2. Unknown Parameter Fusion Identification. As we know, in real applications, the only information we have is the indirect observations $Y^{k}=\left\{y_{k^{\prime}}^{i}, k^{\prime}=1, \ldots, k, i=1, \ldots, M\right\}$ from the $M$ asynchronous sensors, in that, to obtain the fused estimate $\widehat{x}_{k}$ of degradation state according to (13) and (14), we need to estimate unknown parameters $\Theta=$ $\left\{\eta, \sigma^{2}, h^{m}, r^{m}\right\}$ at first. In this paper, the $E M$ algorithm is implemented to identify $\Theta$ based on the online asynchronous sensor observations.
$E M$ algorithm is a widely used iterative algorithm for maximum likelihood parameter estimation with unobserved latent variables. Each iteration of $E M$ algorithm involves two steps: the expectation step (E-step) and the maximization step ( $M$-step). In the $E$-step, a function for the expectation of the log-likelihood is established based on the current estimate of the unknown parameter. In the $M$-step, the parameter estimate is updated through maximizing the expected function found in the E-step, and then the updated parameter estimate is utilized to determine the distribution of the latent variables in the $E$-step of next iteration. By this way, $E M$ algorithm iterates between the two steps until convergence $[14,18]$.

The joint $\log$-likelihood function at time $t_{k}$ is defined as

$$
\begin{aligned}
L_{k}(\Theta) & =\ln p\left(y_{k}, x_{k} \mid \Theta\right) \\
& =\ln p\left(x_{k} \mid \Theta\right)+\ln p\left(y_{k} \mid x_{k}, \Theta\right),
\end{aligned}
$$

where $\left\{y_{k}, x_{k}\right\}$ is the complete data set. Based on the Gauss white noise assumptions, we have $y_{k} \sim N\left(h x_{k}-\eta T_{k} h\right.$, $\left.\Sigma_{k}\left(\sigma^{2}, r^{m}, h^{m}\right)\right)$ and $x_{k} \sim N\left(x_{k-1}+\eta\left(t_{k}-t_{k-2}\right), \sigma^{2}\left(t_{k}-t_{k-1}\right)\right)$; further it follows that

$$
\begin{aligned}
L_{k}(\Theta)= & \ln \left\{\frac{1}{\sqrt{2 \pi \sigma^{2}\left(t_{k}-t_{k-1}\right)}} \exp \left\{-\frac{\left[x_{k}-x_{k-1}-\eta\left(t_{k}-t_{k-1}\right)\right]^{2}}{2 \sigma^{2}\left(t_{k}-t_{k-1}\right)}\right\}\right\} \\
& +\ln \left\{(2 \pi)^{-M / 2}\left|\Sigma_{k}\left(\sigma^{2}, r^{m}, h^{m}\right)\right|^{-1 / 2} \exp \left\{-\frac{1}{2}\left(y_{k}-h x_{k}+\eta T_{k} h\right)^{T}\left(\Sigma_{k}\left(\sigma^{2}, r^{m}, h^{m}\right)\right)^{-1}\left(y_{k}-h x_{k}+\eta T_{k} h\right)^{T}\right\}\right\} \\
= & -\frac{1}{2} \ln \left[2 \pi \sigma^{2}\left(t_{k}-t_{k-1}\right)\right]-\frac{\left[x_{k}-x_{k-1}-\eta\left(t_{k}-t_{k-1}\right)\right]^{2}}{2 \sigma^{2}\left(t_{k}-t_{k-1}\right)}-\frac{M}{2} \ln (2 \pi)-\frac{1}{2} \ln \left|\Sigma_{k}\left(\sigma^{2}, r^{m}, h^{m}\right)\right| \\
& -\frac{1}{2}\left(y_{k}-h x_{k}+\eta T_{k} h\right)^{T}\left(\Sigma_{k}\left(\sigma^{2}, r^{m}, h^{m}\right)\right)^{-1}\left(y_{k}-h x_{k}+\eta T_{k} h\right) .
\end{aligned}
$$


As we said, the EM algorithm consists of two steps: $E$-step and $M$-step in each iteration.

(i) E-step

$$
\begin{aligned}
& \vartheta\left(\Theta \mid \widehat{\Theta}_{k}^{l}\right)=E_{X\left(t_{k}\right) \mid Y^{k}, \widehat{\Theta}_{k}^{(l)}}\left[\ln p\left(y_{k}, x_{k} \mid \Theta\right)\right] \propto-\frac{1}{2} \\
& \cdot \ln \left(\sigma^{2}\right)-\frac{1}{2 \sigma^{2}\left(t_{k}-t_{k-1}\right)}\left[{\widehat{x_{k}^{2}}}^{(l)}+{\widehat{x_{k-1 \mid k}^{2}}}^{(l)}\right. \\
& \quad-2 \widehat{x_{k} x_{k-1 \mid k}}{ }^{(l)}-2 \eta\left(t_{k}-t_{k-1}\right)\left(\widehat{x}_{k}^{(l)}-\widehat{x}_{k-1 \mid k}^{(l)}\right) \\
& \left.+\eta^{2}\left(t_{k}-t_{k-1}\right)^{2}\right]-\frac{1}{2} \ln \left|\Sigma_{k}\left(\sigma^{2}, r^{m}, h^{m}\right)\right| \\
& -\frac{1}{2}\left[y_{k}^{T}\left(\Sigma_{k}\left(\sigma^{2}, r^{m}, h^{m}\right)\right)^{-1} y_{k}\right. \\
& -2 y_{k}^{T}\left(\Sigma_{k}\left(\sigma^{2}, r^{m}, h^{m}\right)\right)^{-1} h \widehat{x}_{k}^{(l)} \\
& +\widehat{x}_{k}^{(l)} h^{T}\left(\Sigma_{k}\left(\sigma^{2}, r^{m}, h^{m}\right)\right)^{-1} h \\
& +\eta h^{T} T_{k}\left(\Sigma_{k}\left(\sigma^{2}, r^{m}, h^{m}\right)\right)^{-1} \\
& \left.+\left(2 y_{k}-2 h \widehat{x}_{k}^{(l)}+\eta T_{k} h\right)\right],
\end{aligned}
$$

where $\widehat{x}_{k}^{(l)}=E\left\{x\left(t_{k}\right) \mid Y^{k}, \widehat{\Theta}_{k}^{(l)}\right\},{\widehat{x_{k}^{2}}}^{(l)}=E\left\{x^{2}\left(t_{k}\right) \mid\right.$ $\left.Y^{k}, \widehat{\Theta}_{k}^{(l)}\right\}=\left(\widehat{x}_{k}^{(l)}\right)^{2}+P_{k}^{(l)}, \widehat{x}_{k-1 \mid k}^{(l)}=E\left\{x\left(t_{k-1}\right) \mid Y^{k}, \widehat{\Theta}_{k}^{(l)}\right\}$, ${\widehat{x_{k-1 \mid k}^{2}}}^{(l)}=E\left\{x^{2}\left(t_{k-1}\right) \mid Y^{k}, \widehat{\Theta}_{k}^{(l)}\right\}=\left(\widehat{x}_{k-1 \mid k}^{(l)}\right)^{2}+P_{k-1 \mid k}^{(l)}$, ${\widehat{x_{k} x_{k-1 \mid k}}}^{(l)}=E\left\{x\left(t_{k}\right) x\left(t_{k-1}\right) \mid Y^{k}, \widehat{\Theta}_{k}^{(l)}\right\}=P_{k, k-1 \mid k}^{(l)}+$ $\widehat{x}_{k-1 \mid k}^{(l)} \widehat{x}_{k}^{(l)}$, and

$$
\begin{aligned}
\widehat{x}_{k-1 \mid k}^{(l)} & =\widehat{x}_{k-1}^{(l)}+\frac{P_{k-1}^{(l)}}{P_{k \mid k-1}^{(l)}}\left(\widehat{x}_{k}^{(l)}-\widehat{x}_{k \mid k-1}^{(l)}\right), \\
P_{k-1 \mid k}^{(l)} & =P_{k-1}^{(l)}-\frac{P_{k-1}^{(l)}}{\left(P_{k \mid k-1}^{(l)}\right)^{2}}\left(P_{k \mid k-1}^{(l)}-P_{k}^{(l)}\right), \\
P_{k, k-1 \mid k}^{(l)} & =\frac{P_{k}^{(l)} P_{k-1}^{(l)}}{P_{k \mid k-1}^{(l)}}
\end{aligned}
$$

where $\hat{x}_{k \mid k-1}^{(l)}, P_{k \mid k-1}^{(l)}, \hat{x}_{k}^{(l)}$, and $P_{k}^{(l)}$ can be calculated from (13) and (14) with $\Theta=\left\{\eta, \sigma^{2}, h^{m}, r^{m}\right\}$ replaced with $\Theta_{k}^{(l)}=\left\{\eta_{k}^{(l)},\left(\sigma^{2}\right)_{k}^{(l)},\left(h^{m}\right)_{k}^{(l)},\left(r^{m}\right)_{k}^{(l)}\right\}$, respectively [24].

(ii) $M$-step: at time $t_{k}$, the estimate of $\Theta$ for the $l+1$ th $E M$ iteration is calculated by

$$
\widehat{\Theta}_{k}^{l+1}=\underset{\Theta}{\arg \max } \vartheta\left(\Theta \mid \widehat{\Theta}_{k}^{l}\right) .
$$

In order to get the value of $\widehat{\Theta}_{k}^{l+1}, G A[26,27]$ is adopted to solve the maximization problem of (20). $G A$ is a heuristic algorithm inspired by the process of natural selection, which is widely used for solving optimization problems. The evolution of $G A$ is an iterative process. It starts with an initial population, usually generated randomly, and the fitness of each individual in the population is evaluated, where the fitness function is the objective function of the optimization problem being solved. The new generation, which is used for the next iteration, is then formed through bioinspired operators, such as selection, mutation, and crossover, based on the fitness. Generally, the algorithm terminates when either a maximum number of generations has been generated or a satisfactory fitness level has been reached for the population.

The iteration of the $E M$ algorithm is terminated if the convergence condition $\left|\widehat{\Theta}^{(l+1)}-\widehat{\Theta}^{(l)} \leq \varsigma\right|$ is satisfied, where $\varsigma$ is a preset threshold.

3.3. RUL Fusion Estimation. From (3), we can see that the RUL is defined as the first passage time of stochastic degradation process $X(t)$. As we know, the first passage time of Wiener process is inverse Gauss distribution, and thus the probability distribution function $f\left(\ell_{k} \mid Y^{k}\right)$ of RUL can be obtained by

$$
\begin{aligned}
& f\left(\ell_{k} \mid Y^{k}\right) \\
& =\frac{\left(\omega-\widehat{x}_{k}\right) \sigma^{2}+P_{k} \eta}{\sqrt{2 \pi\left(P_{k}+\sigma^{2} t_{k}\right)^{3}}} \exp \left\{-\frac{\left(\omega-\widehat{x}_{k}-\eta \ell_{k}\right)^{2}}{2\left(P_{k}+\sigma^{2} \ell_{k}\right)}\right\},
\end{aligned}
$$

where $E\left\{\ell_{k} \mid Y^{k}\right\}=\left(\omega-\widehat{x}_{k}\right) / \eta$ and $\operatorname{var}\left\{\ell_{k} \mid Y^{k}\right\}=((\omega-$ $\left.\left.\widehat{x}_{k}\right) \sigma^{2}+P_{k} \eta\right) / \eta^{3}$.

\section{Simulation Results}

In this section, a simulation example is provided to illustrate the feasibility and effectiveness of the proposed asynchronous RUL fusion estimation algorithm.

The stochastic degradation is formulated by (1) with $\phi=$ $0, \eta=0.3$, and $\sigma^{2}=0.01$. We assume the degradation process is monitored by two asynchronous sensors with sampling periods $T^{1}=0.16$ hours and $T^{2}=0.22$ hours. The fusion period is $T^{f}=0.2$ hours. The observation coefficients of the two sensors are $h^{1}=1.03$ and $h^{2}=0.76$ with measurement noise variances $r^{1}=0.46$ and $r^{2}=0.55$, respectively. Obviously, the two sensors are asynchronous because of different sampling frequencies.

The proposed asynchronous RUL fusion estimation algorithm is used to estimate the RUL of the hidden degradation process. Figure 2 gives the actual degradation path and the estimated one. It can be seen from Figure 2 that the estimated degradation path has good ability to track the actual changes.

In the simulation, the failure threshold $w$ is set to 11.3, and the true failure time (i.e., the first passage time) of the degradation path given in Figure 2 is at 40 hours. To illustrate the effectiveness of the proposed fusion algorithm, 


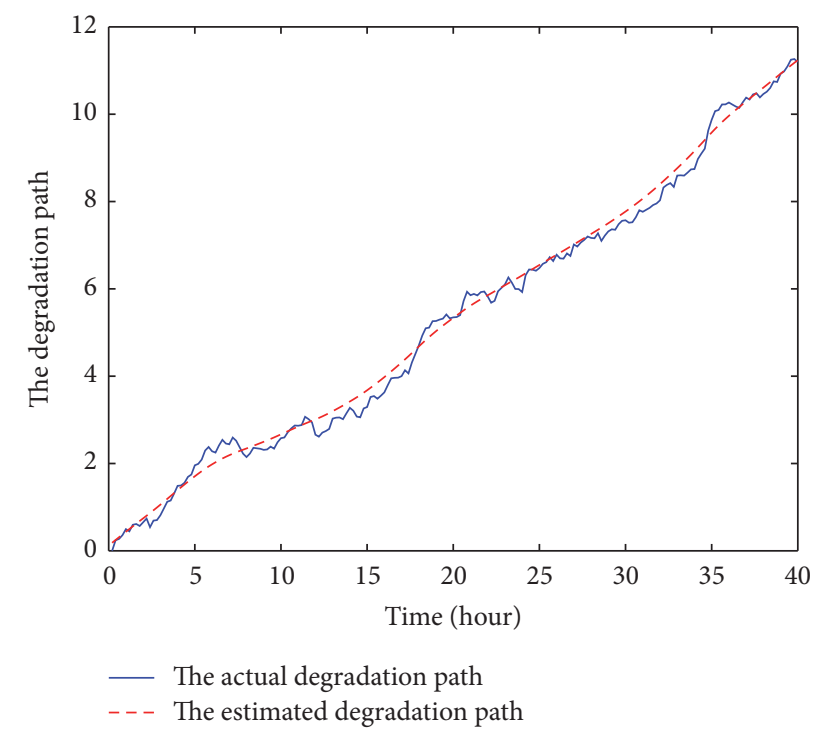

FIgURE 2: The actual degradation path and the estimated.

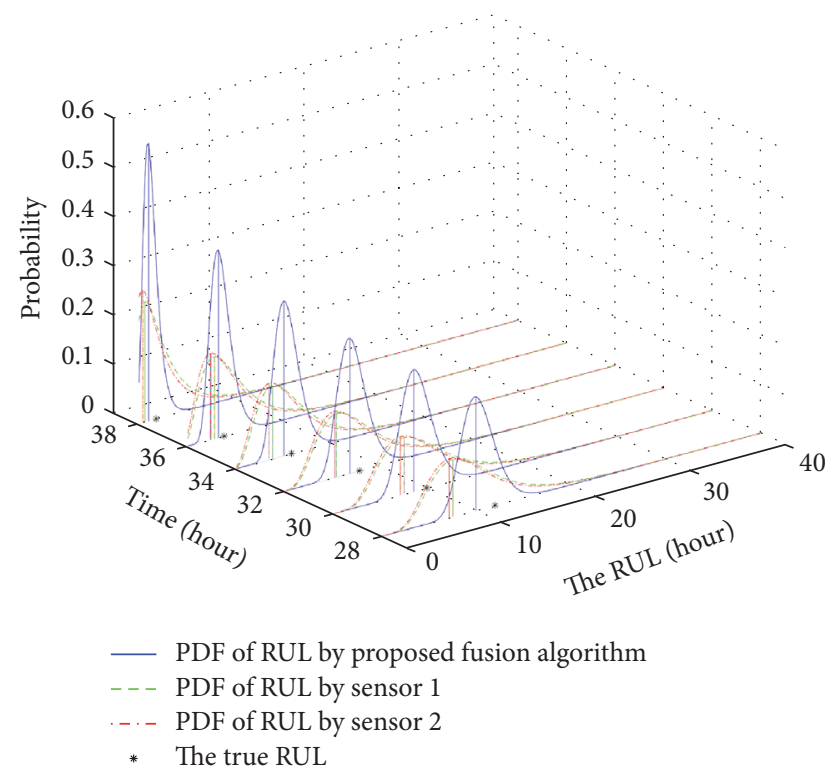

FIgURE 3: The PDFs of RUL at different time instants.

the Probability Distribution Functions (PDFs) of RUL at different time instants are compared in Figure 3, where the PDFs drawn by solid lines are obtained by fusing observations from both of the asynchronous sensors, while the dashed and the dot dashed lines are results of single sensor 1 and sensor 2 , respectively. In order to show this more clearly, the PDFs of RUL at a specific time (36 hours) are also given in Figure 4. It can be seen from Figures 3 and 4 that at each fusion time instant the fused PDF is more concentrated than that of single sensor. Meanwhile, the mean of the fused estimate is nearer to the true RUL (denoted with the asterisk). This means that the fused estimate has smaller estimation error as well as smaller error variation, in other words, better estimation performance, than the estimate obtained from single sensor

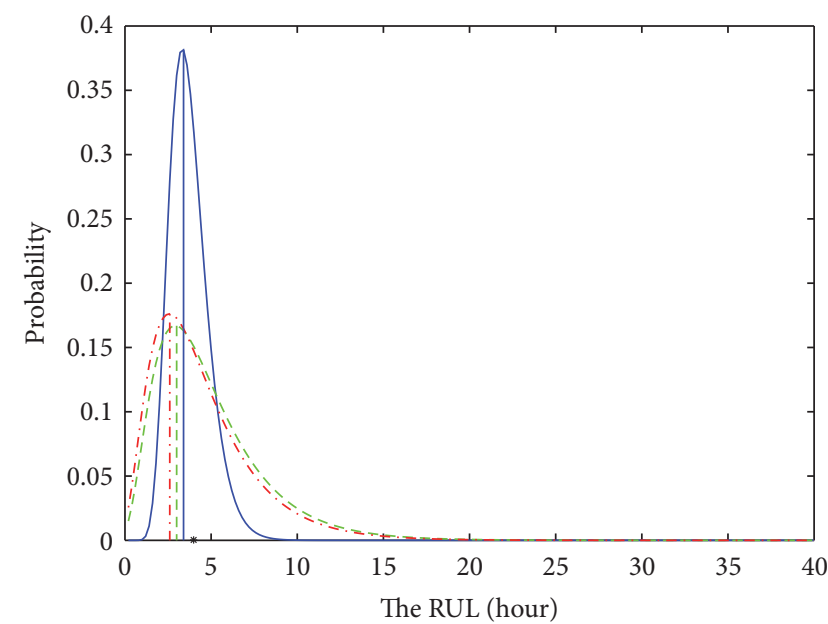

- PDF of RUL by asynchronous sensor fusion
-- PDF of RUL by sensor 1
- - PDF of RUL by sensor 2
* The true RUL

FIgURE 4: The PDFs of RUL at 36 hours.

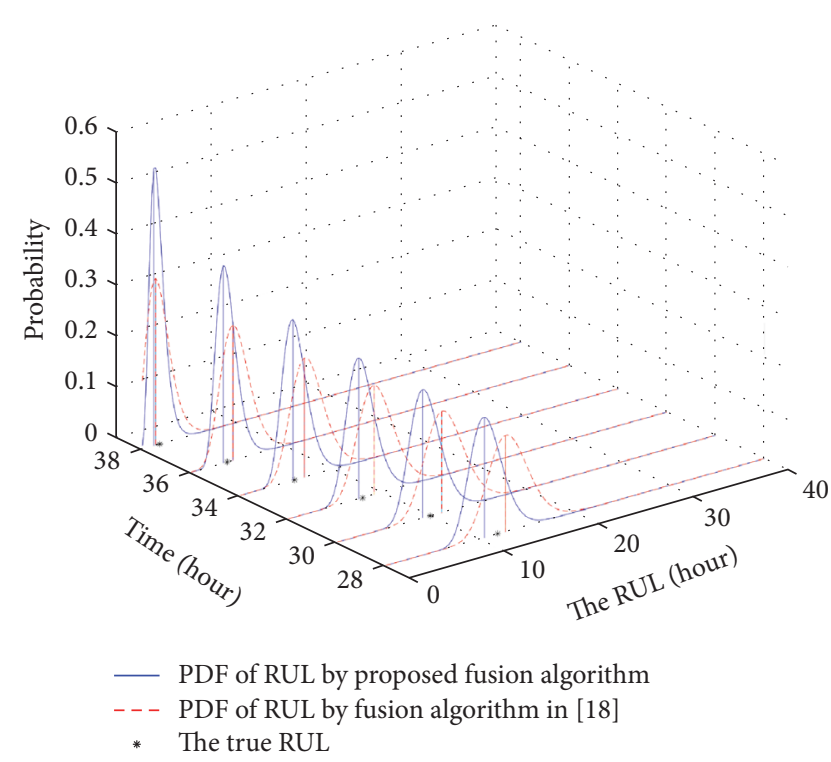

FIGURE 5: Comparison of the proposed algorithm and the approach in [18] under synchronous scenario.

observations. Meanwhile, it can be noticed in Figure 3 that the estimation error decreases with the increase of time.

In addition, it can be seen from Figure 1 that "synchronous" can be regarded as a special case of "asynchronous." Therefore, the proposed asynchronous fusion estimation algorithm is compared with the synchronous fusion estimation method in [18] under synchronous scenario in Figure 5, where $T^{1}=T^{2}=T^{f}=0.2$ hours. From Figure 5, we can see that the proposed algorithm outperforms the fusion algorithm in [18], since it has smaller mean error and more concentrated PDFs of RUL. 


\section{Conclusion}

In this paper, an asynchronous RUL fusion estimation algorithm has been proposed for the latent degradation process with multiple asynchronous monitoring sensors. The asynchronous indirect observations are firstly synchronized to the fusion time and then fused using the Kalman filtering technology to get the estimate of the latent degradation state with the correlations between the involved noise considered and the unknown model parameters identified by the $E M$ and GA. Finally, the fused estimate of the latent degradation state and the updated model parameters are used to get the probability distribution of the RUL. Simulation results demonstrate that fusion of multisource monitoring information from multiple asynchronous sensors can reduce the uncertainty in the systems and that improves the RUL estimation preformation.

Due to the noise correlation introduced by the synchronization process, $G A$ is adopted in this paper to update the estimate of unknown model parameters in the $E M$ algorithm. Further work can focus on deriving an analytical solution by decollating the involved noises.

\section{Conflicts of Interest}

The authors declare that there are no conflicts of interest regarding the publication of this paper.

\section{Acknowledgments}

This work was supported by the National Natural Science Foundation of China under Grants 61304105 and 61520106009 and in part by the Fundamental Research Funds for the Central Universities of China with Grant FRF-TP-16029A3.

\section{References}

[1] X. He, Z. Wang, X. Wang, and D. H. Zhou, "Networked strong tracking filtering with multiple packet dropouts: algorithms and applications," IEEE Transactions on Industrial Electronics, vol. 61, no. 3, pp. 1454-1463, 2014.

[2] A. Heng, S. Zhang, A. C. C. Tan, and J. Mathew, "Rotating machinery prognostics: state of the art, challenges and opportunities," Mechanical Systems and Signal Processing, vol. 23, no. 3, pp. 724-739, 2009.

[3] N. Li, Y. Lei, J. Lin, and S. X. Ding, "An improved exponential model for predicting remaining useful life of rolling element bearings," IEEE Transactions on Industrial Electronics, vol. 62, no. 12, pp. 7762-7773, 2015.

[4] A. K. S. Jardine, D. Lin, and D. Banjevic, "A review on machinery diagnostics and prognostics implementing condition-based maintenance," Mechanical Systems and Signal Processing, vol. 20, no. 7, pp. 1483-1510, 2006.

[5] K. Le Son, M. Fouladirad, A. Barros, E. Levrat, and B. Iung, "Remaining useful life estimation based on stochastic deterioration models: A comparative study," Reliability Engineering and System Safety, vol. 112, pp. 165-175, 2013.

[6] X.-S. Si, W. Wang, C.-H. Hu, and D.-H. Zhou, "Remaining useful life estimation-a review on the statistical data driven approaches," European Journal of Operational Research, vol. 213, no. 1, pp. 1-14, 2011.

[7] R. K. Singleton, E. G. Strangas, and S. Aviyente, "Extended kalman filtering for remaining-useful-life estimation of bearings," IEEE Transactions on Industrial Electronics, vol. 62, no. 3, pp. 1781-1790, 2015.

[8] Y. Qian and R. Yan, "Remaining useful life prediction of rolling bearings using an enhanced particle filter," IEEE Transactions on Instrumentation and Measurement, vol. 64, no. 10, pp. 26962707, 2015.

[9] Z. Liu, G. Sun, S. Bu, J. Han, X. Tang, and M. Pecht, "Particle learning framework for estimating the remaining useful life of lithium-ion batteries," IEEE Transactions on Instrumentation and Measurement, vol. 66, no. 2, pp. 280-293, 2017.

[10] D. Wang, F. Yang, K.-L. Tsui, Q. Zhou, and S. J. Bae, "Remaining useful life prediction of lithium-ion batteries based on spherical cubature particle filter," IEEE Transactions on Instrumentation and Measurement, vol. 65, no. 6, pp. 1282-1291, 2016.

[11] N. Gorjian, Y. Sun, L. Ma, P. Yarlagadda, and M. Mittinty, "Remaining useful life prediction of rotating equipment using covariate-based hazard models - Industry applications," Australian Journal of Mechanical Engineering, pp. 1-10, 2015.

[12] C. Su, Y. Zhang, and H. Zhang, "Product reliability assessment based on proportional hazard degradation model," Journal of Southeast University, vol. 26, no. 3, pp. 480-483, 2010.

[13] Z. Huang, Z. Xu, W. Wang, and Y. Sun, "Remaining useful life prediction for a nonlinear heterogeneous wiener process model with an adaptive drift," IEEE Transactions on Reliability, vol. 64, no. 2, pp. 687-700, 2015.

[14] X.-S. Si, W. Wang, C.-H. Hu, and D.-H. Zhou, "Estimating remaining useful life with three-source variability in degradation modeling," IEEE Transactions on Reliability, vol. 63, no. 1, pp. 167-190, 2014.

[15] R. Edirisinghe, S. Setunge, and G. Zhang, "Application of gamma process for building deterioration prediction," Journal of Performance of Constructed Facilities, vol. 27, no. 6, pp. 763773, 2013.

[16] Z.-Y. Fan, H. Ju, and F.-B. Sun, "Improved gamma process for degradation analysis under nonlinear condition," International Journal of Reliability, Quality and Safety Engineering, vol. 22, no. 6, Article ID 1550030, pp. 267-271, 2015.

[17] K. A. Abaza, "Back-calculation of transition probabilities for Markovian-based pavement performance prediction models," International Journal of Pavement Engineering, vol. 17, no. 3, pp. 253-264, 2016.

[18] M. Wei, M. Chen, and D. Zhou, "Multi-sensor information based remaining useful life prediction with anticipated performance," IEEE Transactions on Reliability, vol. 62, no. 1, pp. 183198, 2013.

[19] M. Dong and D. He, "Hidden semi-Markov model-based methodology for multi-sensor equipment health diagnosis and prognosis," European Journal of Operational Research, vol. 178, no. 3, pp. 858-878, 2007.

[20] Y. Lei, N. Li, and J. Lin, "A new method based on stochastic process models for machine remaining useful life prediction," IEEE Transactions on Instrumentation and Measurement, vol. 65, no. 12, pp. 2671-2684, 2016.

[21] Z. Li and Q. He, "Prediction of railcar remaining useful life by multiple data source fusion," IEEE Transactions on Intelligent Transportation Systems, vol. 16, no. 4, pp. 2226-2235, 2015. 
[22] R. Gravina, P. Alinia, H. Ghasemzadeh, and G. Fortino, "Multisensor fusion in body sensor networks: state-of-the-art and research challenges," Information Fusion, vol. 35, pp. 68-80, 2017.

[23] Y. Hu, Z. Jin, S. Qi, and C. Sun, "Estimation fusion for networked systems with multiple asynchronous sensors and stochastic packet dropouts," Journal of the Franklin Institute. Engineering and Applied Mathematics, vol. 354, no. 1, pp. 145-159, 2017.

[24] D. Simon, Optimal State Estimation: Kalman, Ho, and Nonlinear Approaches, Wiley-Interscience, 2006.

[25] Q. Ge, T. Shao, Z. Duan, and C. Wen, "Performance analysis of the Kalman filter with mismatched noise covariances," IEEE Transactions on Automatic Control, vol. 61, no. 12, pp. 4014-4019, 2016.

[26] T. Baumgartner, I. Chatzigiannakis, S. Fekete, C. Koninis, A. Kroller, and A. Pyrgelis, A Generic Algorithm Library for Heterogeneous Sensor Networks, Springer, Berlin, Germany, 2010.

[27] A. Hore and D. Ziou, "An edge-sensing generic demosaicing algorithm with application to image resampling," IEEE Transactions on Image Processing, vol. 20, no. 11, pp. 3136-3150, 2011. 


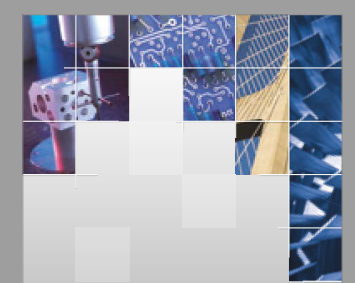

\section{Enfincering}
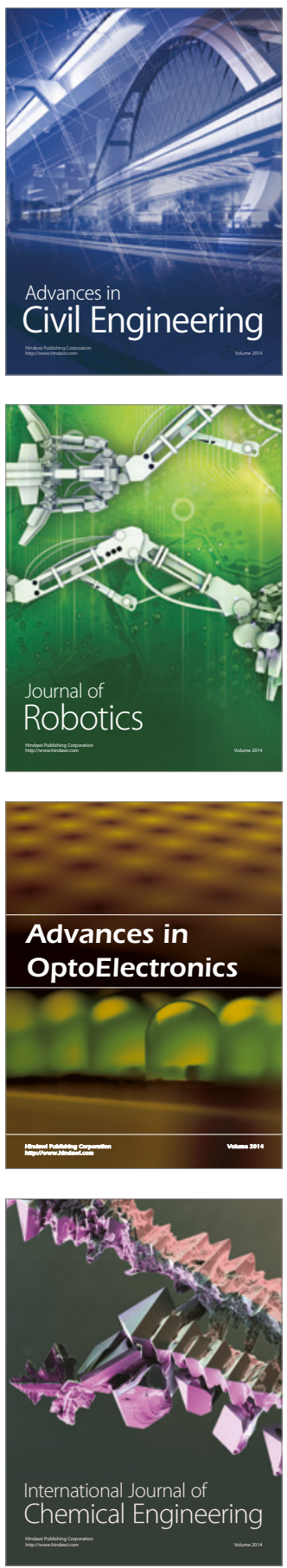

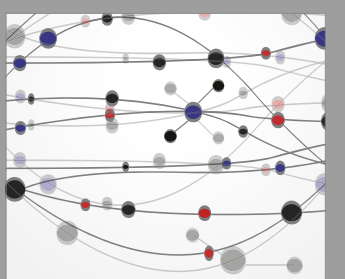

The Scientific World Journal

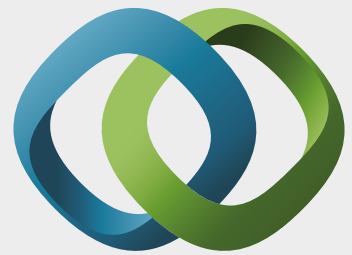

\section{Hindawi}

Submit your manuscripts at

https://www.hindawi.com
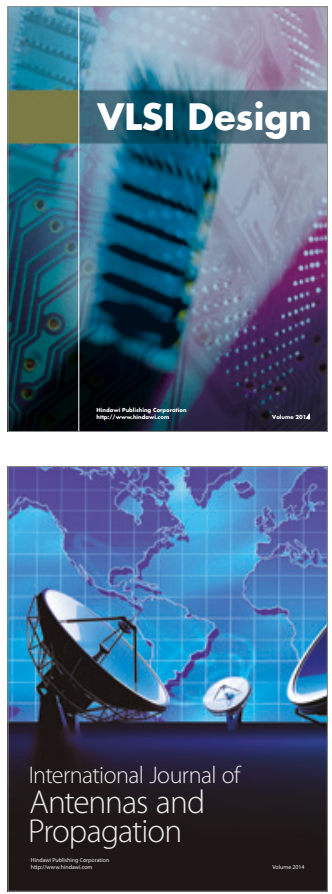

\section{Rotating}

Machinery
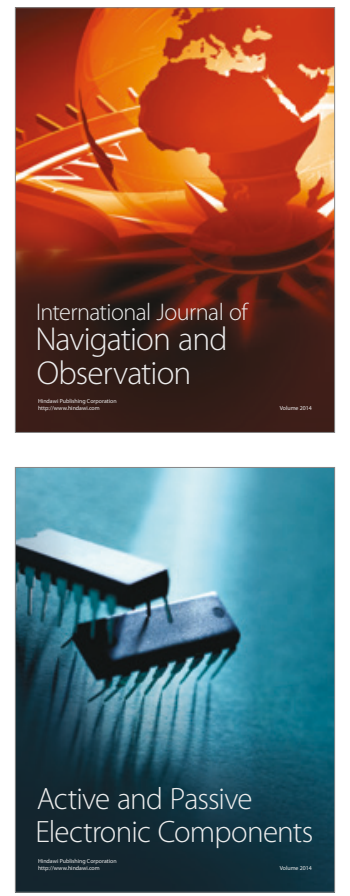
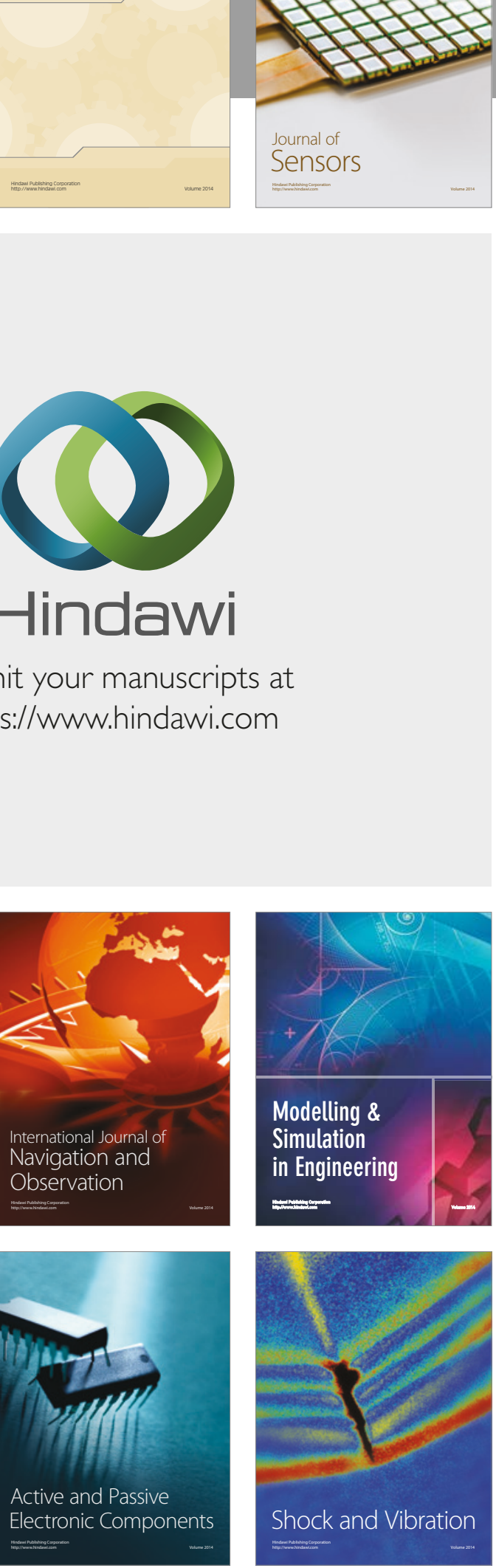
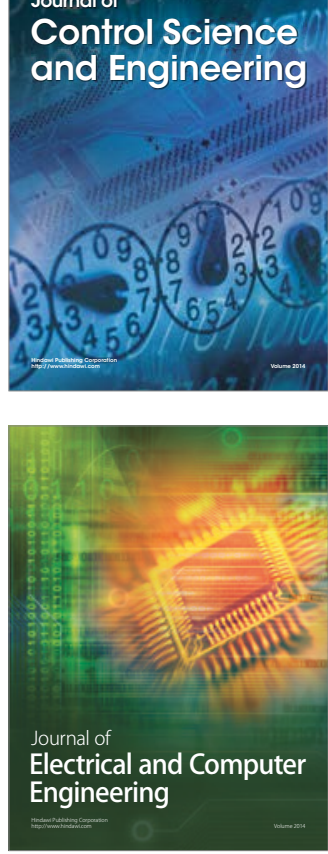

Distributed

Journal of

Control Science

and Engineering
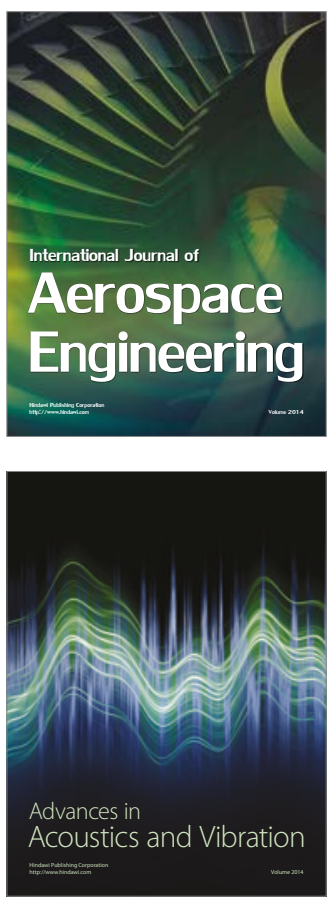

Sensor Networks 